\title{
Optimal Pilot Power Based Channel Estimation Improves the Throughput of Intelligent Reflective Surface Assisted Systems
}

\author{
Jiancheng An, Li Wang, Senior Member, IEEE, Chao Xu, Senior Member, IEEE, Lu Gan, and Lajos Hanzo, \\ Fellow, IEEE
}

\begin{abstract}
Intelligent reflecting surfaces (IRS) have emerged as a promising technology of managing the radio signal propagation by relying on a large number of low-cost passive reflecting elements. In this letter, the optimal pilot power allocation required for accurate channel estimation of IRS-assisted communication systems is investigated. In contrast to conventional channel estimators, where pilot signals are usually designed to be constant-enveloped, we reconsider the pilot design to improve the passive beamforming performance thus resulting in an improved achievable rate. At first sight the result of our analysis appears counter-intuitive, suggesting that at a given total power, more power should be allocated to estimate low-gain channels, since the channel phase impairments are more severe than those of highgain channels. Our simulation results show that when the number of IRS elements is 4 , the rate improvement of our proposed channel estimation scheme over the conventional counterpart may be as high as $25 \%$.
\end{abstract}

Index Terms-Rate improvement, channel estimation errors, intelligent reflecting surface.

\section{INTRODUCTION}

Intelligent reflecting surfaces (IRS) have recently attracted substantial research interests as a benefit of achieving significant cellular coverage, throughput and energy efficiency improvements [1], [2]. Specifically, an IRS is composed of a large number of reconfigurable passive reflecting metasurfaces which are managed by a smart controller to facilitate dynamic adjustments of the signal reflections for enhancing the desired signal power and/or suppressing the interference [3], [4]. In particular, compared to amplify-and-forward relaying, to decode-and-forward relaying and to backscattering-aided communications, a passive IRS reflects and relays signals in a full-duplex manner without incurring self-interference, hence dramatically reducing the energy consumption and hardware

This work was supported in part by the China Scholarship Council. The work of L. Hanzo was supported in part by the Engineering and Physical Sciences Research Council projects EP/ZUO4558/1, EP/ZUO34284/1, and COALESCE, in part by the Royal Societys Global Challenges Research Fund Grant, and in part by the European Research Councils Advanced Fellow Grant QuantCom.

J. An and L. Gan are with the School of Information and Communication Engineering, University of Electronic Science and Technology of China, Chengdu, Sichuan 611731, China. (E-mail: jiancheng_an@163.com; ganlu@uestc.edu.cn).

C. Xu, and L. Hanzo are with the School of Electronics and Computer Science, University of Southampton, SO17 1BJ, UK. (E-mail: cx1g08@soton.ac.uk; 1h@ecs.soton.ac.uk).

L. Wang is with Huawei Technology Sweden R\&D Competence Center, 16994 Stockholm, Sweden. (E-mail: leo.li.wang @ huawei.com). cost by employing cost-efficient passive reflecting components only [5]-[8].

Despite the aforementioned appealing advantages, a pivotal issue in the design of IRS-assisted communication systems is how to tune the reflection elements based on the channel state information (CSI) of all reflecting paths, so that the signals reflected by the IRS can be coherently combined with those arriving via other paths for enhancing the signal power at the target receiver, whilst mitigating the co-channel interference [9]-[11]. The design of passive IRS-aided beamforming has been studied both under continuous and discrete phase shift assumptions concerning the reflecting elements in the context of diverse communication techniques [12]-[15].

Nevertheless, the early constitutions typically assumed having perfect CSI knowledge for all the individual channels between the IRS and the base station (BS) as well as users, which is practically challenging to satisfy, since IRSs are only capable of reflecting the signals but do not have sophisticated channel estimation or other signal processing capability [16][18]. On the other hand, IRSs usually consist of a large number of reflecting elements, which potentially requires a heavy pilot overhead for estimating time-varying channels [17], [19]. In order to alleviate this problem, You et al. [20] divided the IRS elements into several groups, where only the effective channel of the individual group has to be estimated. Naturally, this simplification results in degraded passive IRS-aided beamforming performance, because the reflection coefficients in each group have to be of identical setting based on the limited CSI feedback. Furthermore, Chen et al. [21] modelled the channel estimation problem as a sparse matrix recovery problem, which also significantly reduces the number of pilot symbols required.

However, none of the aforementioned channel estimators considered the effect of channel estimation errors on passive beamforming performance. Furthermore, the pilot signals are usually designed to be constant-enveloped. Against this background, we investigate the optimal power allocation for IRSassisted channel estimation. More explicitly, the contributions of this letter are as follows:

- Firstly, we study the effect of the channel estimation errors on the IRS-aided passive beamforming performance and model the relationship between the pilot power and the achievable rate.

- Secondly, by solving the maximum rate problem, we obtain the optimal pilot design scheme, which some- 


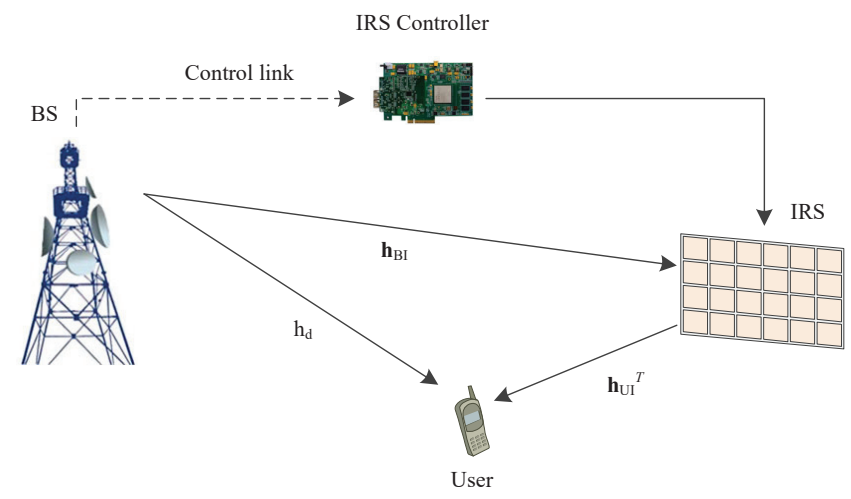

Fig. 1. An IRS-aided point-to-point communication system.

what surprisingly requires us to allocate more power for estimating the low-quality channels. The numerical simulations confirm our analysis.

\section{SySTEM MODEL}

Let us consider the IRS-assisted narrowband TDD system of Fig. 1, where an IRS having $M$ passive reflecting elements is deployed to assist the transmission of a single-antenna BS to a single-antenna user. Each element of the IRS reflects the signal using an individual reflection coefficient, which can be dynamically adjusted by the IRS controller to have the desired signal reflection. Specifically, let $\boldsymbol{\alpha}=\left[\alpha_{1}, \alpha_{2}, \cdots, \alpha_{M}\right]^{T}$ denote the IRS reflection coefficient vector, where $\alpha_{m} \in \mathbb{C}$ denotes the reflection coefficient of the $m$-th IRS reflective element satisfying $\left|\alpha_{m}\right|=1, \forall m=1,2, \cdots, M$, while the phase of each $\alpha_{m}$ can be flexibly adjusted in $[0,2 \pi)$. Let $h_{d}$ denote the direct channel spanning from the BS to the user. Furthermore, let $\mathbf{h}_{B I}$ and $\mathbf{h}_{U I}^{T}$ denote the channels spanning from the BS to the IRS and from the IRS to the user, respectively. Moreover, $h_{d}, \mathbf{h}_{B I}$ and $\mathbf{h}_{U I}$ are assumed to obey the independent and identically distributed (i.i.d.) Rayleigh fading channel model, i.e., $h_{d} \sim \mathcal{C N}\left(0, \beta_{d}\right), \mathbf{h}_{B I} \sim \mathcal{C N}\left(\mathbf{0}, \beta_{B I} \mathbf{I}_{M}\right)$ and $\mathbf{h}_{U I} \sim \mathcal{C N}\left(\mathbf{0}, \beta_{U I} \mathbf{I}_{M}\right)$, where $\beta_{d}, \beta_{B I}$ and $\beta_{U I}$ denote the path loss of $h_{d}, \mathbf{h}_{B I}$ and $\mathbf{h}_{U I}$, respectively.

Given the reflective action of the IRS, the received signal $r_{i}$ of the BS at the $i$-th time instant, is the superposition of the signals from the direct communication link and the reflected links via the IRS, which can be expressed as:

$$
\begin{aligned}
r_{i} & =\left(h_{d}+\mathbf{h}_{U I}^{T} \operatorname{diag}\{\boldsymbol{\alpha}\} \mathbf{h}_{B I}\right) s_{i}+n_{i} \\
& =\left(h_{d}+\boldsymbol{\alpha}^{T} \mathbf{h}_{r}\right) s_{i}+n_{i},
\end{aligned}
$$

where $s_{i}$ denotes the transmit signal and $n_{i} \sim \mathcal{C N}\left(0, \sigma^{2}\right)$ is the additive white Gaussian noise (AWGN) at the $i$-th time instant. Furthermore, $\mathbf{h}_{r}=\mathbf{h}_{B I} \odot \mathbf{h}_{U I}$ is the cascaded reflecting channel via the IRS, where $\odot$ denotes the Hadamard product.

\section{Achievable Rate in the Face of Channel ESTIMATION ERRORS}

In this section, we consider the achievable rate of IRSassisted communication systems in the presence of channel estimation errors. For the sake of illustration, we assume that a total of $(1+M)$ pilots are used for estimating $(1+M)$ channel coefficients, including a direct and $M$ cascaded IRS reflecting channel coefficients. Assuming that the pilot symbol at the $m$-th time slot (TS) is $p_{m}$, we have:

$$
\mathbf{p}=\left[p_{0}, p_{1}, \cdots, p_{M}\right]^{T} .
$$

The simplest 'on-off' strategy of [22] is applied, i.e. at the $m$-th TS, we only activate the $m$-th IRS reflection unit and disable the other reflection units. Specially, at the 0-th TS, all the reflection units are disabled. Since the direct channel is always present, we subtract the influence of the direct channel from $r_{m}, m=1,2, \cdots, M$. Upon collecting the $(1+M)$ residual signals into a vector $\mathbf{u}$, we have

$$
\mathbf{u}=\mathbf{P h}+\mathbf{z},
$$

where $\mathbf{P}=\operatorname{diag}\{\mathbf{p}\}$ denotes the diagonal pilot matrix having $\mathbf{p}$ on its main diagonal, $\mathbf{h}=\left[h_{d}, \mathbf{h}_{r}^{T}\right]^{T}$ represents all the channel coefficients. For the $m$-th element in $\mathbf{u}$ and $\mathbf{z}$, we have $u_{m}=r_{m}-\frac{p_{m} p_{0}^{*}}{\left|p_{0}\right|^{2}} r_{0}$ and $z_{m}=n_{m}-\frac{p_{m} p_{0}^{*}}{\left|p_{0}\right|^{2}} n_{0}$ obeying $z_{m} \sim \mathcal{C N}\left(0, \sigma_{m}^{2}\right)$, where $\sigma_{m}^{2}=\sigma^{2}\left(1+\frac{\left|p_{m}\right|^{2}}{\left|p_{0}\right|^{2}}\right)$. Specially, we have $u_{0}=r_{0}, z_{0}=n_{0}$ and $\sigma_{0}^{2}=\sigma^{2}$ for the direct channel.

Following (3), the least square (LS) channel estimator can be formulated as

$$
\hat{\mathbf{h}}=\mathbf{P}^{-1} \mathbf{u}=\mathbf{h}+\Delta \mathbf{h},
$$

where $\boldsymbol{\Delta} \mathbf{h}=\mathbf{P}^{-1} \mathbf{z}$ is the Gaussian-distributed channel estimation error, i.e. $\boldsymbol{\Delta} \mathbf{h} \sim \mathcal{C N}(\mathbf{0}, \boldsymbol{\Lambda})$, where $\boldsymbol{\Lambda}=$ $\operatorname{diag}\left(\sigma_{0}^{2} /\left|p_{0}\right|^{2}, \sigma_{1}^{2} /\left|p_{1}\right|^{2}, \cdots, \sigma_{M}^{2} /\left|p_{M}\right|^{2}\right)$.

In the conventional channel estimation strategy, the pilot signals are usually designed to have a constant envelope relying on equal pilot power allocation, which results in the same degree of channel estimation error for all paths. Since a low-gain channel is more sensitive to the channel estimation error, the identical power allocation strategy is expected to impose a passive beamforming performance degradation. More specifically, instead of considering the channel estimation only, we directly evaluate the channel estimation performance in terms of the achievable rate of our IRS-assisted system. The achievable rate of IRS-assisted communication systems can be expressed as:

$$
R=\log _{2}\left\{1+\rho\left|h_{d}+\boldsymbol{\alpha}^{T} \mathbf{h}_{r}\right|^{2}\right\},
$$

where $\rho=P / \sigma^{2}$ is the signal-to-noise ratio and $P$ is the average transmit signal power.

Using the estimated channel coefficients, the optimal IRS reflecting coefficients satisfying (5) are given by [3]

$$
\alpha_{m}=\frac{\hat{h}_{d} \hat{h}_{r, m}^{*}}{\left|\hat{h}_{d} \hat{h}_{r, m}^{*}\right|}, \quad \forall m=1,2, \cdots, M,
$$

where $\hat{h}_{d}$ and $\hat{h}_{r, m}$ denote the estimated direct channel coefficient and the estimated $m$-th cascaded reflecting channel coefficient of the IRS, respectively.

Upon substituting (6) into (5), the expression of $R$ is formulated in (7), where $(a)$ holds since the channel estimation error 


$$
\begin{aligned}
& R=\log _{2}\left\{1+\rho\left|h_{d}+\sum_{m=1}^{M} \frac{\hat{h}_{d} \hat{h}_{r, m}^{*}}{\left|\hat{h}_{d} \hat{h}_{r, m}^{*}\right|} h_{r, m}\right|^{2}\right\} \\
& \quad \stackrel{(a)}{\simeq} \log _{2}\left\{1+\rho\left|h_{d}+\sum_{m=1}^{M} \frac{\left(h_{d}+\Delta h_{d}\right)\left(h_{r, m}+\Delta h_{r, m}\right)^{*}}{\left|h_{d} h_{r, m}^{*}\right|} h_{r, m}\right|^{2}\right\} \\
& \quad \stackrel{(b)}{\simeq} \log _{2}\left\{1+\rho\left|h+\sum_{m=1}^{M} \frac{h_{r, m} h_{r, m}^{*} \Delta h_{d}+h_{d} h_{r, m} \Delta h_{r, m}^{*}}{\left|h_{d} h_{r, m}^{*}\right|}\right|^{2}\right\},
\end{aligned}
$$

Low SNR: $\mathcal{E}\{R\}=\mathcal{E}\left\{\log _{2}\left\{1+\rho\left|h+\sum_{m=1}^{M} \frac{h_{r, m} h_{r, m}^{*} \Delta h_{d}+h_{d} h_{r, m} \Delta h_{r, m}^{*}}{\left|h_{d} h_{r, m}^{*}\right|}\right|^{2}\right\}\right\}$

$$
\begin{aligned}
& \stackrel{(c)}{=} \frac{1}{\ln 2}\left\{1+\rho \mathcal{E}\left\{\mid h+\sum_{m=1}^{M} \frac{h_{r, m} h_{r, m}^{*} \Delta h_{d}+h_{d} h_{r, m} \Delta h_{r, m}^{*} \mid}{\left|h_{d} h_{r, m}^{*}\right|}\right\}\right\} \\
& \stackrel{(d)}{=} \frac{1}{\ln 2}\left\{1+\rho \mathcal{E}\left\{|h|^{2}+\left|\sum_{m=1}^{M} \frac{h_{r, m} h_{r, m}^{*} \Delta h_{d}+h_{d} h_{r, m} \Delta h_{r, m}^{*}}{\left|h_{d} h_{r, m}^{*}\right|}\right|^{2}\right\}\right\} \\
& \stackrel{(e)}{=} \frac{1}{\ln 2}\left\{1+\rho \mathcal{E}\left\{|h|^{2}\right\}+\rho M \mathcal{E}\left\{\left|\frac{\left.\left.\left.h_{r, m} h_{r, m}^{*} \Delta h_{d}\right|^{2}\right\}+\rho M \mathcal{E}\left\{\left|\frac{h_{d} h_{r, m} \Delta h_{r, m}^{*}}{\left|h_{d} h_{r, m}^{*}\right|}\right|^{2}\right\}\right\}}{\left|h_{d} h_{r, m}^{*}\right|}\right|\right\}\right. \\
& =\frac{1}{\ln 2}\left\{1+\rho \mathcal{E}\left\{|h|^{2}\right\}+\rho M \mathcal{E}\left\{\frac{\left|h_{r, m}\right|^{2}}{\left|h_{d}\right|^{2}}\left|\Delta h_{d}\right|^{2}\right\}+\rho M \mathcal{E}\left\{\left|\Delta h_{r, m}\right|^{2}\right\}\right\} \\
& \stackrel{(f)}{=} \frac{1}{\ln 2}\left\{1+\rho\left\{\beta_{d}+M \beta_{r}+\frac{M \pi^{3 / 2}}{4} \sqrt{\beta_{d} \beta_{r}}+\frac{M(M-1) \pi^{2}}{16} \beta_{r}\right\}+\rho M \frac{\beta_{r}}{\beta_{d}} \frac{\sigma^{2}}{\left|p_{0}\right|^{2}}+\rho M \frac{\sigma^{2}\left(1+\frac{|p|^{2}}{\left|p_{0}\right|^{2}}\right)}{|p|^{2}}\right\} \\
& =\frac{1}{\ln 2}\left\{1+\rho\left\{\beta_{d}+M \beta_{r}+\frac{M \pi^{3 / 2}}{4} \sqrt{\beta_{d} \beta_{r}}+\frac{M(M-1) \pi^{2}}{16} \beta_{r}\right\}+\frac{\rho M \sigma^{2}}{\beta_{d}}\left\{\frac{\beta_{r}+\beta_{d}}{\left|p_{0}\right|^{2}}+\frac{\beta_{d}}{|p|^{2}}\right\}\right\},
\end{aligned}
$$

$\operatorname{High} S N R: \quad \mathcal{E}\{R\} \simeq \log _{2}\left\{\rho\left\{\beta_{d}+M \beta_{r}+\frac{M \pi^{3 / 2}}{4} \sqrt{\beta_{d} \beta_{r}}+\frac{M(M-1) \pi^{2}}{16} \beta_{r}\right\}+\frac{\rho M \sigma^{2}}{\beta_{d}}\left(\frac{\beta_{r}+\beta_{d}}{\left|p_{0}\right|^{2}}+\frac{\beta_{d}}{|p|^{2}}\right)\right\}$

is generally small, while $(b)$ holds since we omit the secondorder term of the channel estimation error. Furthermore, $\Delta h_{d}$ and $\Delta h_{r, m}$ denote the channel estimation error related to the direct channel and to the $m$-th cascaded reflecting channel satisfying $\Delta h_{d} \sim \mathcal{C N}\left(0, \frac{\sigma_{0}^{2}}{\left|p_{0}\right|^{2}}\right)$ and $\Delta h_{r, m} \sim \mathcal{C N}\left(0, \frac{\sigma_{m}^{2}}{\left|p_{m}\right|^{2}}\right)$, respectively, while $h=h_{d}+\sum_{m=1}^{M} \frac{h_{d} h_{r, m}^{*}}{\left|h_{d} h_{r, m}^{*}\right|} h_{r, m}$ represents the uncontaminated channel gain in the absence of channel estimation errors.

In order to determine the influence of channel estimation errors on the achievable rate, the ergodic capacity of IRS-assisted communication systems is formulated at low SNR as in (8), where $(c)$ is the equivalent form of the logarithmic function at low SNR [23], $(d)$ and $(e)$ hold since the mean of the cross terms in the first and second equation of (8) is $0 ;(f)$ holds since $h_{d}, h_{r, m}, \Delta h_{d}$, and $\Delta h_{r, m}$ are independent of each other, remembering that we have $\sigma_{m}^{2}=\sigma^{2}\left(1+\frac{\left|p_{m}\right|^{2}}{\left|p_{0}\right|^{2}}\right)$. In $(f)$, an identical power is allocated for all pilots used for estimating the IRS channels, i.e., $|p|^{2}=\left|p_{1}\right|^{2}=\left|p_{2}\right|^{2}=\cdots=\left|p_{M}\right|^{2}$, since they experience the same path loss having identical second-order statistics. The proof of the equivalent expression of $\mathcal{E}\left\{|h|^{2}\right\}$ in $(f)$ is shown in Appendix A. Following the same philosophy, the approximate ergodic capacity at high SNR is shown in (9).

Remark 1: Here, we only consider a single IRS scenario, where the same power is assigned to all the pilots for estimating the IRS's reflected channels. When a more sophisticated distributed IRS system is considered, the assumption in (8) and (9) should be generalized to the case, where the same power is assigned to the pilots used for estimating the channels reflected by the same IRS. By contrast, different power should be assigned to the pilots employed for estimating the channels reflected by different IRSs.

Since the ergodic rate $\mathcal{E}\{R\}$ in (8) and (9) has a direct 


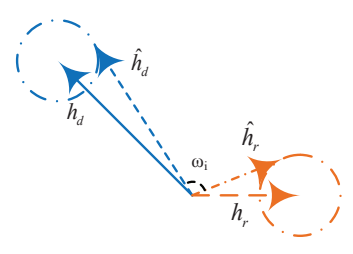

(a) Identical power allocation

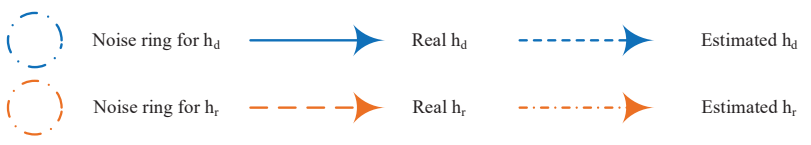

Fig. 2. The phase of the estimated IRS reflecting coefficient under two different pilot power allocation schemes. It shows that estimating a more accurate weak channel helps to obtain a more accurate reflecting coefficient.

relationship with the pilot power used for channel estimation, we aim for finding an optimal pilot power allocation scheme for maximizing $\mathcal{E}\{R\}$ of (8) and (9) under a total power constraint, which is formulated as:

$$
\begin{array}{cc}
\max & \mathcal{E}\{R\} \\
\text { subject to } & \left|p_{0}\right|^{2}+M|p|^{2}=P_{t},
\end{array}
$$

where $P_{t}$ is the total power available at the transmitter.

\section{The Optimal Pilot Design Scheme}

In this section, we conceive our optimal pilot design scheme for channel estimation for maximizing the ergodic achievable rate. Note that $\mathcal{E}\{R\}$ has the same optimization metric at low SNR and high SNR. More specifically, the problem in (10) can be simplified to

$$
\begin{array}{cc}
\max & \frac{\beta_{r}+\beta_{d}}{\left|p_{0}\right|^{2}}+\frac{\beta_{d}}{|p|^{2}} \\
\text { subject to } & \left|p_{0}\right|^{2}+M|p|^{2}=P_{t},
\end{array}
$$

where the constant term in $\mathcal{E}\{R\}$ of (8) has been omitted. The pilot power allocation problem in (11) then leads to:

$$
|p|^{2}=\frac{1}{M+\sqrt{M(1+\eta)}} P_{t}
$$

and

$$
\left|p_{0}\right|^{2}=\frac{\sqrt{M(1+\eta)}}{M+\sqrt{M(1+\eta)}} P_{t},
$$

where we have $\eta=\frac{\beta_{r}}{\beta_{d}}$, indicating that the optimal power allocation scheme depends on the quotient of the pass loss of the reflecting channel and the direct channel, which is available at the BS for controlling the link margin.

It is observed from (12) and (13) that the power $|p|^{2}$ of the pilot allocated for estimating the reflected channels decreases with $\eta$, while the opposite is true for $\left|p_{0}\right|^{2}$. This indicates that when the path loss of the reflected channel or the direct channel is high, we should allocate more power to the pilots used for estimating these severely attenuated channels

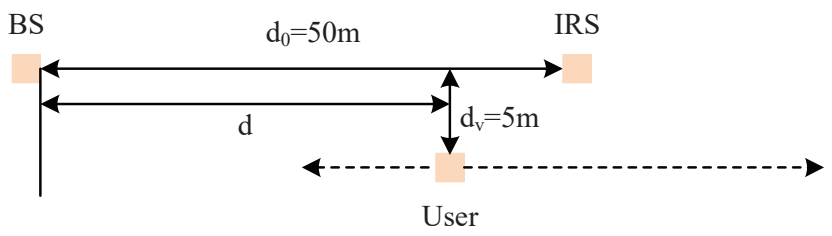

Fig. 3. Simulation scenario 1: a single user is stationary on a line parallel to the IRS-BS line.

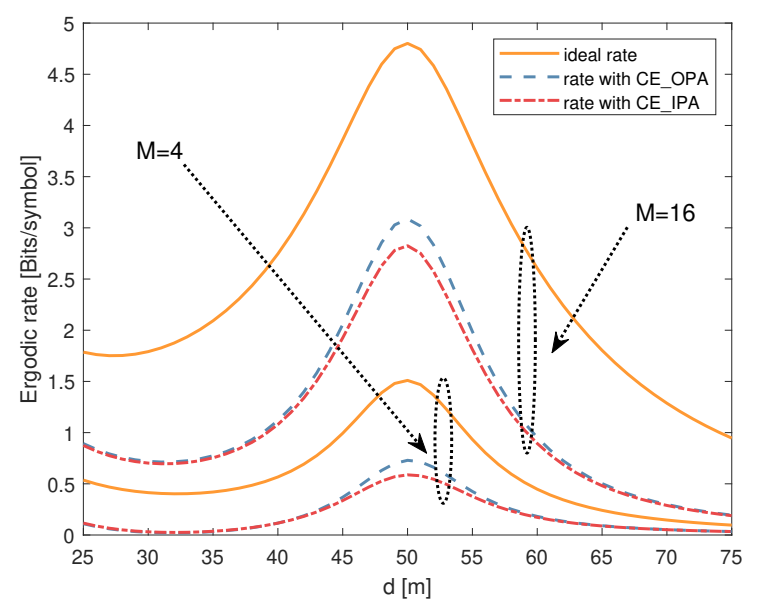

Fig. 4. Rate comparison in the face of channel estimation errors under simulation scenario 1, where 'CE_OPA' represents the proposed optimal power allocation scheme, while 'CE_IPA' represents the conventional identical power allocation scheme.

more accurately for attaining improved passive beamforming performance. On the other hand, for the pilot used for estimating the reflective path, we have $\frac{M+\sqrt{M(1+\eta)}}{M+1} \rightarrow 1$ with the increase of $M$, which implies that the proposed power allocation scheme is equivalent to the identical power allocation scheme, when $M$ is large. Although this means that the rate improvement becomes limited for large $M$, our algorithm does have a significant performance improvement under low to moderate number of IRS elements. Nonetheless there are many beneficial application scenarios, such as indoor environments [24].

A simple schematic diagram is portrayed in Fig. 2 for better understanding (12) and (13). For the sake of simplicity, we assume having a single IRS reflecting element, i.e. $M=1$. More explicitly, we assume $\beta_{d}>\beta_{r}$, and denote the phase of the reflecting coefficient $\alpha$ by $\omega$. By comparing Fig. 2 (a) and (b), it can be readily seen that the optimized power allocation scheme makes the estimated $\omega_{o}$ more similar to the real $\omega$ by estimating a more accurate $h_{r}$, since the phase dispersion is more severe for a low-gain channel.

\section{Simulation Results}

In this section, we provide numerical simulation results for verifying the benefits of our proposed pilot power allocation scheme for channel estimation in IRS-assisted communication systems. The path loss functions of $h_{d}, \mathbf{h}_{U I}$ and $\mathbf{h}_{B I}$ are modeled by $\beta_{d}=\beta_{0}\left(d_{U B} / d_{0}\right)^{-\alpha_{U B}}, \beta_{U I}=\beta_{0}\left(d_{U I} / d_{0}\right)^{-\alpha_{U I}}$ and 


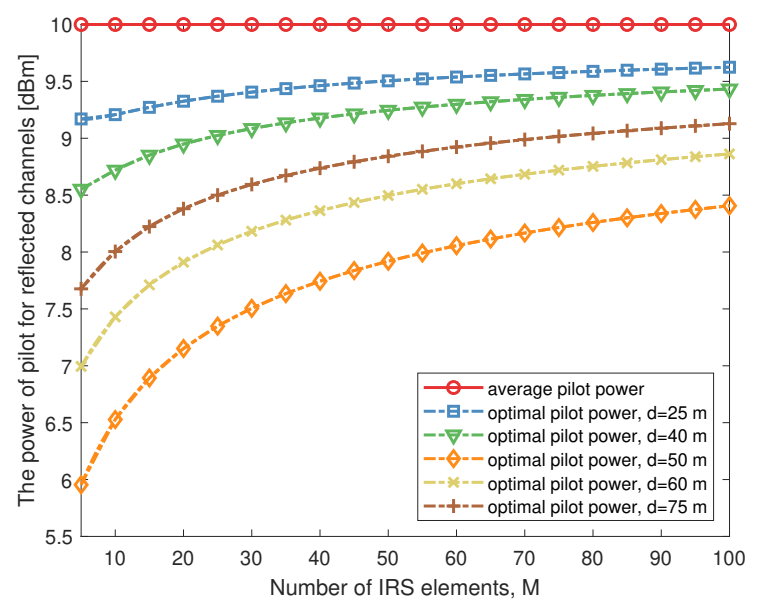

Fig. 5. The power of pilot for estimating the reflected channels versus the number of IRS elements.

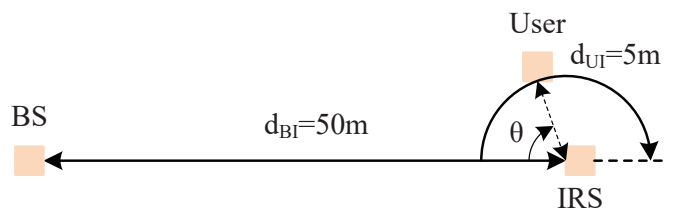

Fig. 6. Simulation scenario 2: a single user is positioned on a semicircle near the IRS.

$\beta_{B I}=\beta_{0}\left(d_{B I} / d_{0}\right)^{-\alpha_{B I}}$, respectively, where $d_{0}=1$ meter (m) denotes the reference distance, $\beta_{0}=-20 \mathrm{~dB}$ represents the path loss at the reference distance, $d_{U B}, d_{U I}$, and $d_{B I}$ denote the distance between the user and BS, between the user and the IRS, as well as between the IRS and the BS, respectively, while $\alpha_{U B}, \alpha_{U I}$ and $\alpha_{B I}$ denote the path loss factors for $h_{d}, \mathbf{h}_{U I}$ and $\mathbf{h}_{B I}$, respectively. Due to the relatively long distance and random scattering effect between the BS and the user, we set $\alpha_{U B}=4.8, \alpha_{U I}=2.1$, and $\alpha_{B I}=2$ in the numerical examples, which is in line with [3], [25]. Moreover, the average transmit power of users is $P=20 \mathrm{dBm}$, while the power of the noise is assumed to be $\sigma^{2}=-60 \mathrm{dBm}$. The total power available at the transmitter is $P_{t}=P(M+1)$.

Since the authors of [3], [25] have reported the rate-benefits of users near the IRS, we first consider a single-user system, as shown in Fig. 3. The vertical distance between the user and IRS-BS line is $d_{v}=5 \mathrm{~m}$, while the distance between the BS and IRS is set to $d_{B I}=50 \mathrm{~m}$. Let us denote the horizontal distance between the BS and the user by $d \mathrm{~m}$. Then we have $d_{U B}=\sqrt{d^{2}+25}$ and $d_{U I}=\sqrt{(50-d)^{2}+25}$. In Fig. 4, we compare the achievable rate of our proposed optimal power allocation scheme and of the conventional identical power allocation scheme, where the legend 'CE_OPA' and 'CE_IPA' represent the achievable rate of the optimal and of the identical pilot power allocation scheme, respectively, while the legend 'ideal rate' represents the achievable rate without channel estimation errors. Observed from Fig. 4 that as expected, a user near the IRS achieves a higher rate than users

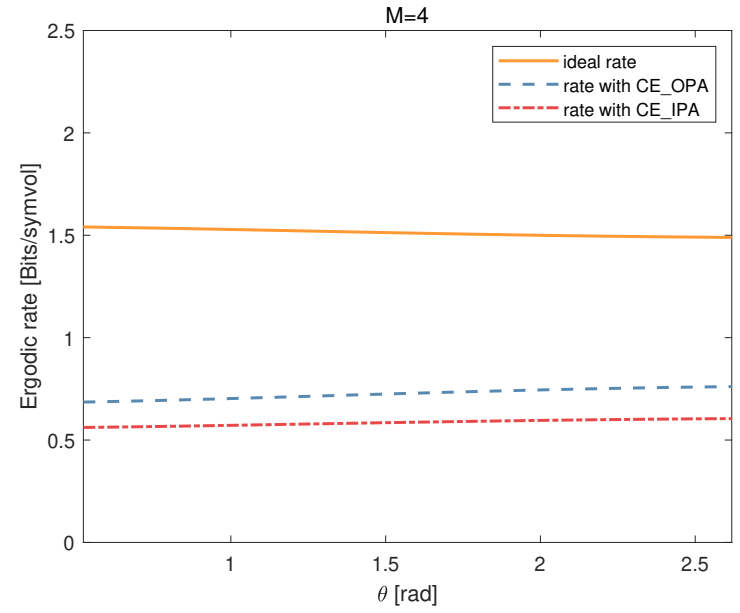

Fig. 7. Rate comparison with channel estimation errors under simulation scenario 2, where 'CE_OPA' represents the proposed optimal power allocation scheme, while 'CE_IPA' represents the conventional identical power allocation scheme.

located far away from both the BS and the IRS. Naturally, in case of realistic channel estimation errors, the rate attained is degraded. However, our proposed pilot power allocation scheme mitigates this degradation by allocating more power to the weaker channel. When the number of IRS elements is $M=4$ at $d=50 \mathrm{~m}$, the proposed pilot power allocation scheme improves the achievable rate as high as $25 \%$, from about 0.6 recorded for the conventional pilot power allocation scheme to about 0.75 .

To get some insight into the proposed power allocation scheme, Fig. 5 shows the power of pilot used for estimating the reflected channels versus the number of IRS elements $M$, where the average pilot power is $10 \mathrm{dBm}$. It can be seen from Fig. 5 that with the increase of $M$, the power of pilot used for estimating the reflected channels becomes closer to the average power, which is consistent with our analysis. On the other hand, Fig. 5 also demonstrates that as the user moves towards the IRS, the proposed power allocation scheme and the conventional scheme will behave more differently, which further confirms the conclusion in Fig. 4.

Next, we consider the scenario shown in Fig. 6 to verify the performance improvement versus user directions [3]. Since we have seen in Fig. 4 that the user has a good performance improvement near the IRS, the user is positioned on a circle having a radius of $d_{U I}=5 \mathrm{~m}$. The distance between the IRS and BS is set to $d_{B I}=50 \mathrm{~m}$. Let $\theta$ denote the angle between the user-IRS and BS-IRS lines, then we have $d_{U B}=$ $\sqrt{(50-5 \cos \theta)^{2}+25 \sin ^{2} \theta}$. Furthermore, the number of IRS elements is set to $M=4$. The simulation results are shown in Fig. 7, where the legends have the same meaning as in Fig. 4. It can be seen from Fig. 7 that the proposed pilot power allocation scheme provides a substantial rate improvement over the identical pilot power allocation scheme.

\section{Conclusions}

In this letter, we studied the effect of channel estimation on the passive beamforming performance of IRS-assisted commu- 
nication systems. The optimal pilot design scheme is found by establishing the relationship between the pilot power and the achievable rate of IRS-assisted communication systems. By allocating higher pilot power to the weaker channels, the IRS's passive beamforming performance is improved compared to that of the conventional identical power allocation scheme, thus improving the achievable rate, especially for the scenario with a small to moderate number of IRS elements, such as indoor environments. In this compact letter, we only analyzed the IRS-aided SISO system. In multi-user/distributedIRS aided communication scenarios, the power allocation between multiple users and IRSs requires further research for exploiting the full advantage of the IRSs.

\section{APPENDIX A \\ PROOF OF $(f)$ IN $(8)$}

Remembering that $h_{d}, h_{B I, m} \quad h_{U I, m}$ are Gaussiandistributed and independent of each other, we have

$$
\begin{aligned}
\mathcal{E}\left\{\left|h_{r, m}\right|\right\} & =\mathcal{E}\left\{\left|h_{B I, m}\right|\right\} \mathcal{E}\left\{\left|h_{U I, m}\right|\right\} \\
& =\frac{\pi}{4} \sqrt{\beta_{B I} \beta_{U I}}=\frac{\pi}{4} \sqrt{\beta_{r}},
\end{aligned}
$$

and

$$
\begin{aligned}
\mathcal{E}\left\{\left|h_{r, m}\right|^{2}\right\} & =\mathcal{E}\left\{\left|h_{B I, m}\right|^{2}\right\} \mathcal{E}\left\{\left|h_{U I, m}\right|^{2}\right\} \\
& =\beta_{B I} \beta_{U I}=\beta_{r} .
\end{aligned}
$$

Hence, the expression of $\mathcal{E}\left\{|h|^{2}\right\}$ can be expanded as

$$
\begin{aligned}
\mathcal{E}\left\{|h|^{2}\right\} & =\mathcal{E}\left\{\left|h_{d}+\sum_{m=1}^{M} \frac{h_{d} h_{r, m}^{*}}{h_{d} h_{r, m}^{*} \mid} h_{r, m}\right|^{2}\right\} \\
& =\mathcal{E}\left\{\left(\left|h_{d}\right|+\sum_{m=1}^{M}\left|h_{r, m}\right|\right)^{2}\right\} \\
& =\mathcal{E}\left\{\left|h_{d}\right|^{2}\right\}+\mathcal{E}\left\{\sum_{m=1}^{M}\left|h_{r, m}\right|^{2}\right\} \\
& +\mathcal{E}\left\{2\left|h_{d}\right| \cdot \sum_{m=1}^{M}\left|h_{r, m}\right|\right\} \\
& +\mathcal{E}\left\{\sum_{m_{1}=1}^{M} \sum_{m_{2}=1, m_{2} \neq m_{1}}^{M}\left|h_{r, m_{1}}\right| \cdot\left|h_{r, m_{2}}\right|\right\} \\
& =\beta_{d}+M \beta_{r}+\frac{M \pi^{3 / 2}}{4} \sqrt{\beta_{d} \beta_{r}}+\frac{M(M-1) \pi^{2}}{16} \beta_{r},
\end{aligned}
$$

where $\mathcal{E}\{|a|\}=\frac{\sqrt{\pi}}{2} \sigma_{a}$ and $\mathcal{E}\left\{|a|^{2}\right\}=\sigma_{a}^{2}$ for a complex Gaussian random variable $a \sim \mathcal{C N}\left(0, \sigma_{a}^{2}\right)$.

\section{REFERENCES}

[1] Q. Wu and R. Zhang, "Towards smart and reconfigurable environment: Intelligent reflecting surface aided wireless network," IEEE Communications Magazine, 2019 in press.

[2] H. Zhang, B. Di, L. Song, and Z. Han, "Reconfigurable intelligent surfaces assisted communications with limited phase shifts: How many phase shifts are enough?," IEEE Transactions on Vehicular Technology, 2020 in press.
[3] Q. Wu and R. Zhang, "Intelligent reflecting surface enhanced wireless network via joint active and passive beamforming," IEEE Transactions on Wireless Communications, vol. 18, no. 11, pp. 5394-5409, 2019.

[4] B. Di, H. Zhang, L. Li, L. Song, Y. Li, and Z. Han, "Practical hybrid beamforming with limited-resolution phase shifters for reconfigurable intelligent surface based multi-user communications," IEEE Transactions on Vehicular Technology, 2020 in press.

[5] C. Huang, A. Zappone, G. C. Alexandropoulos, M. Debbah, and C. Yuen, "Reconfigurable intelligent surfaces for energy efficiency in wireless communication," IEEE Transactions on Wireless Communications, vol. 18, no. 8, pp. 4157-4170, 2019.

[6] Z. Zhou, N. Ge, W. Liu, and Z. Wang, "RIS-aided offshore communications with adaptive beamforming and service time allocation," arXiv preprint arXiv:1911.03240, 2019.

[7] Z. Zhou, N. Ge, Z. Wang, and L. Hanzo, "Joint transmit precoding and reconfigurable intelligent surface phase adjustment: A decompositionaided channel estimation approach," 2019 in press.

[8] S. Gopi, S. Kalyani, and L. Hanzo, "Intelligent reflecting surface assisted beam index-modulation for millimeter wave communication," arXiv preprint arXiv:2003.12049, 2020.

[9] E. Basar, "Reconfigurable intelligent surface-based index modulation: A new beyond MIMO paradigm for 6G," IEEE Transactions on Communications, 2020 in press.

[10] B. Zheng, Q. Wu, and R. Zhang, "Intelligent reflecting surface-assisted multiple access with user pairing: NOMA or OMA?," IEEE Communications Letters, 2020 in press.

[11] E. Björnson, Ö. Özdogan, and E. G. Larsson, "Intelligent reflecting surface vs. decode-and-forward: How large surfaces are needed to beat relaying?," IEEE Wireless Communications Letters, 2019 in press.

[12] T. Bai, C. Pan, Y. Deng, M. Elkashlan, and A. Nallanathan, "Latency minimization for intelligent reflecting surface aided mobile edge computing," arXiv preprint arXiv:1910.07990, 2019.

[13] T. Hou, Y. Liu, Z. Song, X. Sun, Y. Chen, and L. Hanzo, "MIMO assisted networks relying on large intelligent surfaces: A stochastic geometry model," arXiv preprint arXiv:1910.00959, 2019.

[14] C. Pan, H. Ren, K. Wang, M. Elkashlan, A. Nallanathan, J. Wang, and L. Hanzo, "Intelligent reflecting surface enhanced MIMO broadcasting for simultaneous wireless information and power transfer," arXiv preprint arXiv:1908.04863, 2019.

[15] C. Pan, H. Ren, K. Wang, W. Xu, M. Elkashlan, A. Nallanathan, and L. Hanzo, "Multicell MIMO communications relying on intelligent reflecting surface," [Online] https://arxiv. org/abs/1907.10864, 2019.

[16] Y. Han, W. Tang, S. Jin, C. Wen, and X. Ma, "Large intelligent surfaceassisted wireless communication exploiting statistical CSI," IEEE Transactions on Vehicular Technology, vol. 68, no. 8, pp. 8238-8242, 2019.

[17] B. Zheng and R. Zhang, "Intelligent reflecting surface-enhanced OFDM: Channel estimation and reflection optimization," IEEE Wireless Coтmunications Letters, 2019 in press.

[18] Z. Wang, L. Liu, and S. Cui, "Channel estimation for intelligent reflecting surface assisted multiuser communications: Framework, algorithms, and analysis," arXiv preprint arXiv:1912.11783, 2019.

[19] T. L. Jensen and E. De Carvalho, "On optimal channel estimation scheme for intelligent reflecting surfaces based on a minimum variance unbiased estimator," arXiv preprint arXiv:1909.09440, 2019.

[20] C. You, B. Zheng, and R. Zhang, "Progressive channel estimation and passive beamforming for intelligent reflecting surface with discrete phase shifts," arXiv preprint arXiv:1912.10646, 2019.

[21] J. Chen, Y. Liang, H. Cheng, and W. Yu, "Channel estimation for reconfigurable intelligent surface aided multi-user MIMO systems," arXiv preprint arXiv:1912.03619, 2019.

[22] Y. Yang, B. Zheng, S. Zhang, and R. Zhang, "Intelligent reflecting surface meets OFDM: Protocol design and rate maximization," arXiv preprint arXiv:1906.09956, 2019.

[23] D. Tse and P. Viswanath, Fundamentals of wireless communication. Cambridge university press, 2005.

[24] S. Gong, X. Lu, D. Hoang, D. Niyato, L. Shu, D. Kim, and Y. Liang, "Towards smart radio environment for wireless communications via intelligent reflecting surfaces: A comprehensive survey," arXiv preprint arXiv:1912.07794, 2019.

[25] S. Zhang and R. Zhang, "Capacity characterization for intelligent reflecting surface aided MIMO communication," arXiv preprint arXiv:1910.01573, 2019. 